\title{
A Wireless IoT System Towards Gait Detection in Stroke Patients
}

\author{
Swetha Atnoor, Dr. Mohammad Abdul Waheed \\ VTU CPGS Kalaburagi
}

\begin{abstract}
For this project we wanted to create smart shoe that can give the user feedback on any recognized walking abnormalities as fast as possible. Even though we think this will be more helpful for older users, the product will be useful for people of all ages with disabilities in walking and seeing.

Using the concept of the internet of things (IoT), to detect and translate physical data from real world, the shoe will be able to provide real time results from the user's walking. Automatic fall detection systems represent a modern answer to some of these problems, research about systems for the automatic detection of falls gained momentum. In these devices, the technology is used to detect if a fall occurs and an alarm is triggered without human intervention. These systems would help to reduce the time of arrival of medical caregiver, and accordingly to decrease the mortality rate.
\end{abstract}

Keywords: IoT, Smart shoe, Heart Stroke.

\section{INTRODUCTION}

By developing a smart-shoe that can track weight distribution and abnormal walking patterns the user would be able to see the data for themselves through the application. The shoe will track the user's walking patterns and over the course of a few minutes be able to give the user different types of feedback, from the type of support the user needs, to ways to improve on their walking patterns every day. For example, if the application sees that the user puts more weight on their heels, a daily recommendation could be to try and put a little more weight at the top off the foot when walking. This sort of shoe will have the potential to help many different types of people who have these chronic foot issues.

There is a need to design and build a wearable technology system, which isn't bulky and dissipates very little power in the biomedical vital parameter monitoring so that the location of patient can be tracked in real time when he is unwell. Such system can monitor the sensor data and upload it to cloud over internet and also capable of taking some curial decision using the IoT. Problems are what kind of technology would be used so that the above mentioned problem will be overcome easily and accurately.

\section{LITERATURE REVIEW}

It has now become an important concern for medical awareness and the growth of wireless mobile technology. For this reason, ubiquitous healthcare solutions have become important as they provide services anytime, anywhere. In order to accomplish our needs, Android smartphone devices have observed and analyzed the Ecs [ECG] by the fourth mobile monitoring terminal. Because of the use of wireless sensor networks in healthcare, we can reduce the complications of wired networks and we can move healthcare from one location to another. The mobile phone is used as a barcode decoder for medical care as an extension of the monitoring scheme. In order to provide better and more comprehensive medical services. We can use barcode decoders to validate and assist patients during drug management.

$>$ Dementia can be characterized as a dynamic issue influencing the sensory system that prompts the advancement of psychological deformities. It is viewed as dynamic and hopeless, and in the eighteenth century it was characterized as an absence of limit.

> According to Cole (2012), the World Health Organization characterizes dementia as "a cerebrum malady, typically an endless or dynamic illness, which incorporates clutters of memory, considering, introduction, and understanding and other progressed cortical capacities, computation, and learning. Capacity, dialect and judgment. "

$>$ This is before healthcare providers and researchers realize that this is due to brain degeneration.

Dementia has many different aspects and it is difficult to diagnose and determine the specific cause. The only way to definitively diagnose dementia is to complete the autopsy of the brain on deceased individuals suffering from the disease. Dementia is a huge financial health issue.

\section{PROBLEM STATEMENT}

In the existing study a care taker is to be employed for the patients suffering from dementia continuously which is not feasible in the real time. The care taker may fall asleep or may have gone out somewhere during that time the dementia patients may go to unknown place or may fall down and cause lot of damage to the head,. By the time the care taker comes back there may be lot of loss of blood from the head which may cause death the patient.

\section{PROPOSED WORK}

The proposed system helps people with dementia in many ways. Patients with dementia must be given extra care. We are designing a model that includes a sensor that can be used very easily to identify dementia patients with reduced physical posture. It shows the patient's exact mark. The proposed system tracks the current location if the dementia patient is missed an alert SMS is sent to 
the care taker with the exactly location of the patient. And the proposed system also deals with the fall detection of the patients suffering from dementia. If the patient falls immediately an alert SMS is sent to the care taker s well as the physician.

When the tag signal drops, the wireless sensor network should signal the user either because the patient has moved to a hard-to-reach location or the tag's battery has died. The system should be able to infer patterns of behavior over time and be able to compare patterns of behavior obtained at different time intervals.

\section{RESULTS}

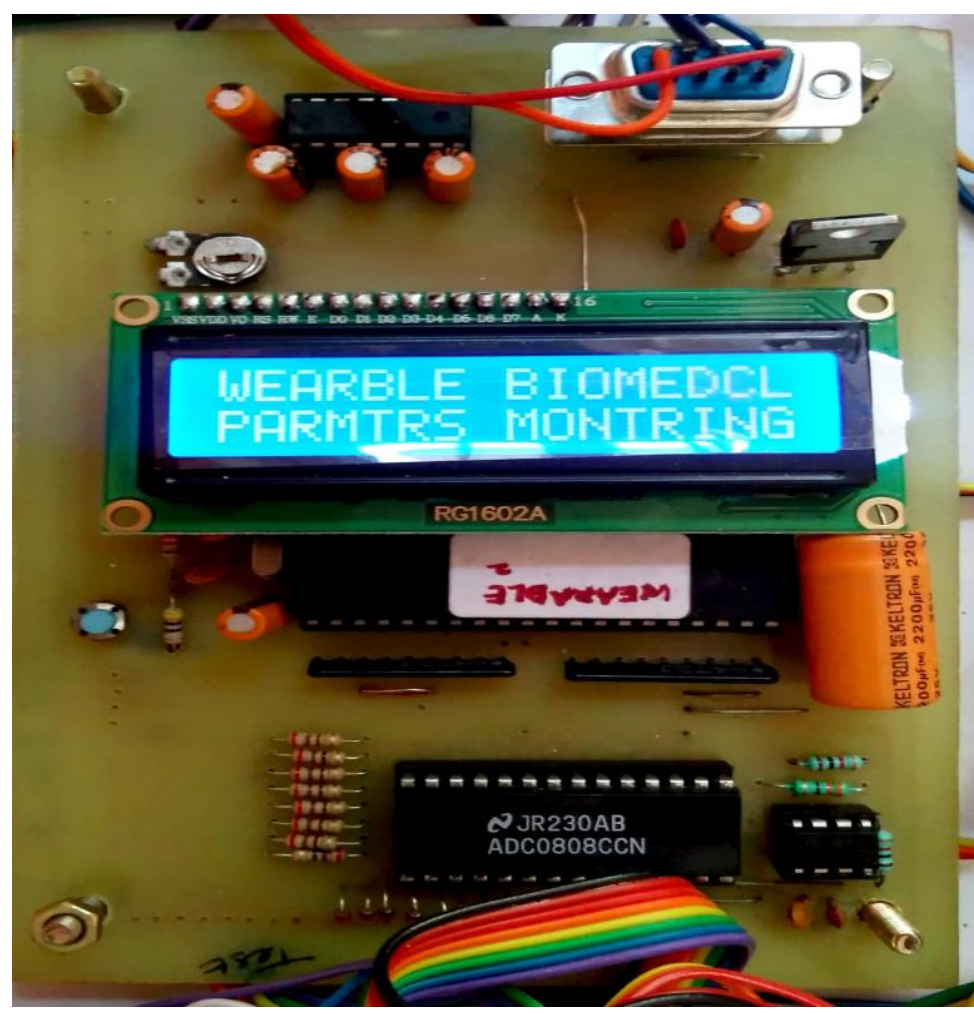

Fig 1. Over all module

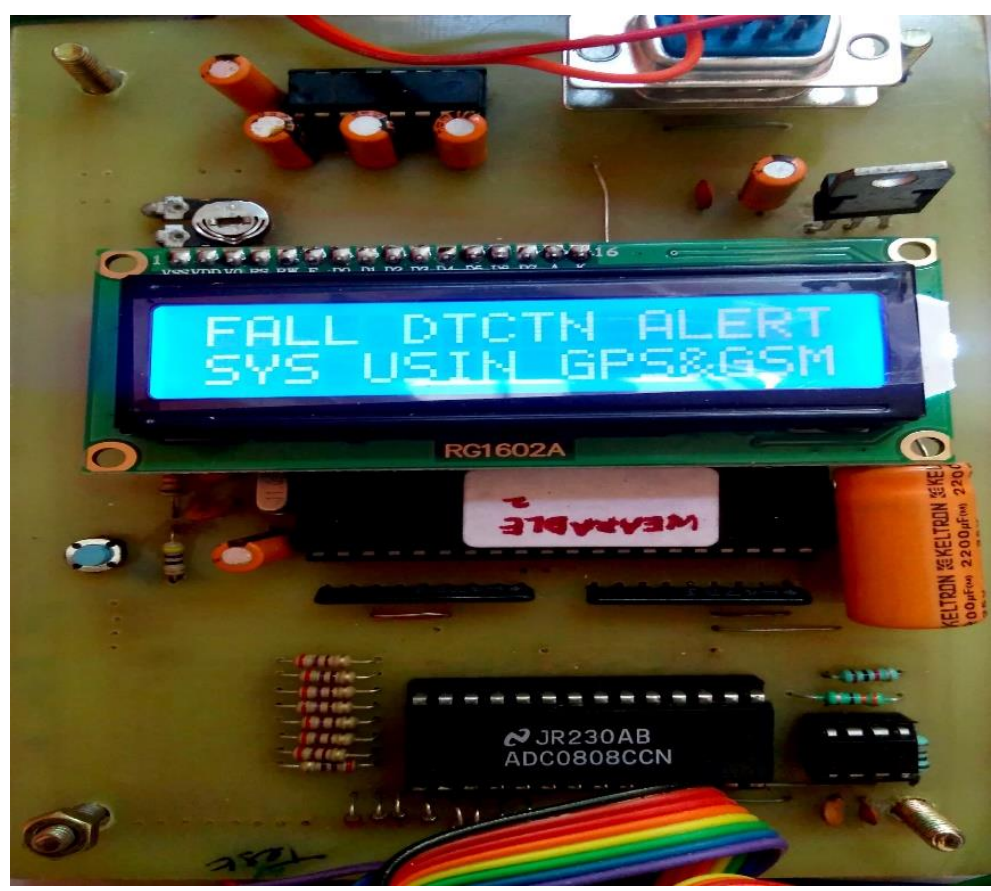

Fig 2. Fall detection alert 


\section{CONCLUSION}

The aim of current project was to build a shoe device which tracks the current location of the patients. As stated in the introduction section dementia means loss of memory therefore it is important to track the location of the patients suffering from dementia. And the other objective of the current project was to identify the fall detection of the patient. If patient has fallen down due to some reason the device detects it and sends an alert SMS to the care taker and the physician.

\section{REFERENCES}

[1] Mike Addle see, Rupert Curwen, Steve Hodges, Joe Newman, Pete Stages, Andy Ward, and Andy Hopper. Implementing a sentient computing system. IEEE Computer Magazine, 34:50-56, 2001.

[2] Rakesh Agrawal and Ramakrishnan Srikanth. Mining sequential patterns. In Proc. of the Int'1 Con-fence on Data Engineering (ICDE), pages 3-14, 1995.

[3] Autoid.org. Active and passive rfid: Two distinct, but complementary, technologies for real-time supply chain visibility.

[4] Christopher M. Bishop. Pattern Recognition and Machine Learning (Information Science and Statistics). Springer, August 2006.

[5] M Boustani, B Peterson, L Hanson, R Harris, and C Karson. Screening for dementia. A systematic evidence review. Rockville, MD: Agency for Healthcare Research and Quality.

[6] Malaz Boustani, Britt Peterson, Laura Hanson, Russell Harris, and Kathleen N. Lahr. Screening for Dementia in Primary Care: A Summary of the Evidence for the U.S. Preventive Services Task Force. Annals of Internal Medicine, 138(11):927-937, June 2003.

[7] Henry Brodaty, Brian M. Draper, and Lee-Fay Low. Behavioural and psychological symptoms of dementia: a seven-tiered model of service delivery. Medical journal of Australia, 178(5):231-234, 2003.

[8] Barry Brumitt, John Krumm, B. Meyers, and S. Shafer. Ubiquitous computing and the role of ge-ometry. IEEE Personal Communications, 7:41-43, October 2000. 